\title{
Lipid Interference in the Determination of the Concentration of Haemoglobin in Plasma Using the ACA SX Analyzer ${ }^{1}$ )
}

\author{
Christian Heller', Rolf Hinzmann' ${ }^{2}$, Michael Hofinann ${ }^{1.3}$, Silke Kaufhold ${ }^{1}$, Eber'hard Henkel ${ }^{2}$ and \\ Michael Oellerich ${ }^{1}$
}
' Abteilung Klinische Chemie, Zentrallabor, Zentrum Innere Medizin, Georg-August-Universität Göttingen, Göttingen, Germany
2 Institut für Klinische Chemie II der Medizinischen Hochschule Hannover im Krankenhaus Oststadt, Hannover, Germany
${ }^{3}$ Max-Planck-Institut für Biophysikalische Chemie, Göttingen, Germany

Summary: In comparison to a triple wavelength procedure, the dual wavelength method for the determination of plasma haemoglobin concentration using the ACA analyzer showed considerable interference with hypertriglyceridaemic (triacylglycerols $>2.3 \mathrm{mmol} / \mathrm{l}$ ) plasma. By addition of isolated human lipoprotein fractions to normotriglyceridaemic plasma, chylomicrons were identified as a major source of interference with the ACA plasma haemoglobin method, whereas VLDL was without effect up to a triacylglycerol concentration of $5.7 \mathrm{mmol} / \mathrm{l}$. Airfuge ultracentrifugation proved to be a reliable means for removal of interfering lipid. We conclude that the extent of lipid interference with the ACA plasma haemoglobin method is highly dependent on the type of lipoprotein present. An accurate measurement of plasma haemoglobin concentrations in non-fasting plasma can only be ensured after lipid removal through airfuge ultracentrifugation.

\section{Introduction}

Plasma haemoglobin can be quantified on the ACA (Du Pont) analyzer using a dual wavelength method. The original application of Golf, Schneider; Friemann et al. (1) involved measuring the haemoglobin absorbance at $540 \mathrm{~nm}$ and correcting for background absorption at 600 $\mathrm{nm}$. Recently, the manufacturer modified the procedure to make use of the potentially more specific measurement with filter $9(577 \mathrm{~nm})$ in combination with filter $10(600 \mathrm{~nm})(2)$.

Bilirubin, triacylglycerols, carboxyhaemoglobin and myoglobin were investigated by the manufacturer as potential causes of interference. In the case of plasma triacylglycerol it was claimed that concentrations up to $442.5 \mathrm{mg} / \mathrm{dl}(5.0 \mathrm{mmol} / \mathrm{l})$ yield an interference below $10 \%$. Since it is well known that clinical chemical assays based on photometric absorption measurements can be affected by hyperlipidaemia (3) we have studied in detail the nature and extent of lipid interference on the determination of plasma haemoglobin concentrations using the ACA SX plasma haemoglobin method and have compared the results with those from an established triple wavelength method (4).

\footnotetext{
1) This investigation was supported by Du Pont de Nemours (Deutschland) GmbH, Du Pont Straße 1, D-61343 Bad Homburg, Germany.
}

\section{Materials and Methods}

Routine patient blood samples $(n=32)$ were collected into plastic tubes containing ammonium heparinate (S-Monovette $5.5 \mathrm{ml}$, Sarstedt, post box 1220, D-51582 Nümbrecht, Germany). Plasma was separated by low speed centrifugation at $3000 \mathrm{~g}$ for $10 \mathrm{~min}$.

\section{Photometric methods}

Comparison method

The triple wavelength comparison method was carried out using a DU-7500 photometer (Beckman Instruments GmbH, Frankfurter Ring 115, D-80807 München, Germany) with automatic calculation of concentration values. Undiluted plasma samples were measured simultaneously at the main wavelength of $578 \mathrm{~nm}$ and the two subtraction wavelengths of 598 and $562 \mathrm{~nm}$. The haemoglobin concentration was calculated according to the method of Kahn et al. (5) using the respective factors published by Fairbanks et al. (4).

$\mathrm{Hb}=1000 \cdot\left[1.550 \cdot \mathrm{A}_{578}-0.689 \cdot \mathrm{A}_{598}-0.861 \cdot \mathrm{A}_{562}\right] \mathrm{mg} / \mathrm{l}$.

When absorbance values $>2.0$ occurred, the sample was diluted with 2 volumes of isotonic $\mathrm{NaCl}$ and results were multiplied by 3 .

\section{Test method}

The dual wavelength method for measuring plasma haemoglobin concentration on the ACA SX analyzer (Du Pont) was performed according to the manufacturer's instructions. Filter $9(577 \mathrm{~nm})$ was used for the main wavelength and filter $10(600 \mathrm{~nm})$ for the reference wavelength (2). Determinations were performed as single measurements.

\section{Calibration of the test method}

ACA result calculation was defined by the use of calibration factors, with $\mathrm{CO}$ and $\mathrm{Cl}$ representing the instrument coefficients 
for slope and intercept in linear calibration procedurcs. For calibration of the dual wavelength haemoglobin method, these factors have to be set by the following procedure:

1. Enter coefficients for $\mathrm{C} 0$ and $\mathrm{Cl}$ (for option, enter the values as specified in the appendix).

2. Freshly collect at least 10 clear patient heparinate plasma samples (triacylglyccrol concentrations $<2.3 \mathrm{mmol} / \mathrm{l}$ ) with plasma haemoglobin values assigned from the triple wavelength method. Triple wavelength haemoglobin values should be in the concentration range between 300 and $1200 \mathrm{mg} / \mathrm{l}$ for these samples. Apply these samples to the ACA measurement in an undiluted fashion.

3. Apply the non-parametric linear regression procedure (6) to obtain slope $b$ and intercept $a$ of the concentration data for these samples, with the triple wavelength method values entered as $x-$ values and the values of the ACA method as $y$-values. For the procedure to be applicable, the correlation coefficient $r$ is expected to yield a value $>0.990$.

4. Modify the calibration coefficients $\mathrm{CO}$ and $\mathrm{Cl}$ of the ACA according to the following calculation formulae: $\mathrm{CO}_{\text {new }}=\mathrm{CO}_{\text {old }}-\mathrm{a}$; $\mathrm{Cl}_{\text {new }}=\mathrm{Cl}_{\text {old }} / \mathrm{b}$. Enter these values using mode 41 of the ACA analyzer.

Example: Initial $\mathrm{C} 0=-7.47$, initial $\mathrm{Cl}=-27.79$.

a (obtained from regression analysis ) $=2.0, b=1.057$.

Result (to be entered in mode 41 of the ACA instrument):

$$
\begin{aligned}
& \mathrm{CO}_{\text {new }}=\mathrm{CO}_{\text {old }}-\mathrm{a}=-7.47-2.0=-9.47 \\
& \mathrm{Cl}_{\text {new }}=\mathrm{Cl}_{\text {old }} \mathrm{b}=-27.79 / 1.057=-26.29 .
\end{aligned}
$$

\section{Performance control}

Instrument performance was monitored with the $\mathrm{CoSO}_{4}$ absorbance test solution supplied by the instrument manufacturer. Performing this dual-wavelength procedure as recommended, we obtained a between-days coefficient of variation of $0.4 \%(n=21)$. With an in-house plasma-derived precision control of $324 \mathrm{mg} / \mathrm{l}$ plasma haemoglobin, the between-days coefficient of variation was $2.1 \%$ $(\mathrm{n}=21)$.

\section{Human chylomicron and VLDL preparation}

A pool of fresh lipaemic human plasma from blood donors was used for preparation of human chylomicron and VLDL fractions. Purification was achieved by fractionation according to particle density. One volume of plasma was carefully overlayered with one volume of $9 \mathrm{~g} / \mathrm{l} \mathrm{NaCl}, 0.2 \mathrm{~g} / \mathrm{l} \mathrm{NaN} 3,1 \mathrm{mmol} / 1$ EDTA. After centrifugation at $30000 \mathrm{~g}$ for $2 \mathrm{~h}$ at $15^{\circ} \mathrm{C}$ the pale yellow supernatant $\left(\mathrm{S}_{\mathrm{f}}>400\right.$, predominantly chylomicrons) was recovered by tube slicing. The infranatant was again overlayered with an equal volume of $9 \mathrm{~g} / \mathrm{NaCl}, 0.2 \mathrm{~g} / \mathrm{l} \mathrm{NaN}, 1 \mathrm{mmol} / \mathrm{l}$ EDTA and recentrifuged for $16 \mathrm{~h}$ at $10^{\circ} \mathrm{C}$ and a relative centrifuge force of $110000 \mathrm{~g}$ to yield the lipoprotein fraction $S_{f} 20-400$ (i.e. predominantly VLDL).

Both fractions were recentrifuged at their respective density for $16 \mathrm{~h}$ at $110000 \mathrm{~g}$ and $10^{\circ} \mathrm{C}$ and the supernatants recovered by tube slicing. These preparations were then used for the lipid interference measurements. Concentrations of triacylglycerols and cholesterol were $24.1 \mathrm{mmol} / \mathrm{l}$ triacylglycerols and $12.2 \mathrm{mmol} / \mathrm{l}$ cholesterol in the chylomicron fraction and $21.2 \mathrm{mmol} / \mathrm{l}$ triacylglycerols and 16.8 $\mathrm{mmol} / \mathrm{l}$ cholesterol in the VLDL fraction. The size of the lipoprotein particles was determined with a NICOMP 70 laser particle sizer (Particle Analytic Meßgeräte $\mathrm{GmbH}$, Olpener Straße 150, D-50933 Köln, Germany).

\section{Other procedures}

Lipaemic plasma samples were clarified by flotation of triacylglycerol-rich lipoproteins using an air-driven ultracentrifuge (Airfuge, rotor A100/30, both from Beckman Instruments $\mathrm{GmbH}$, Frankfurter Ring 115, D-80807 München, Germany). For each sample, two tubes were filled with $175 \mu \mathrm{l}$ of plasma and centrifuged for 20 min at $160000 \mathrm{~g}$ yielding ca. $250 \mu \mathrm{l}$ of clear delipidated plasma for $\mathrm{Hb}$ determination in an ACA micro-cup.

Haemolysates were prepared by addition of a 10-fold excess of cold $\mathrm{Na}_{2} \mathrm{CO}_{3}$-solution $(0.1 \mathrm{~g} / \mathrm{l})$ to EDTA-anticoagulated human blood samples. After an incubation of $30 \mathrm{~min}$, the solution was sonified with a tip sonicator (Branson Ultrasonic S. A., Chemin du Faubourg-de-Cruseilles 9, CH-1228 Carouge-Geneve, Switzerland) set at medium performance for $3 \times 20 \mathrm{~s}$ and then filtered through a $0.2 \mu \mathrm{m}$ ultrafilter (Schleicher and Schuell, Hahnestraße 3, D-37586 Dassel, Germany).

Triacylglycerol and cholesterol concentrations were determined on a Hitachi 747 random access clinical chemical analyzer (Hitachi Ltd. Instrument Division, 882, Ichige, Kațsuta-shi, Ibaraki-ken, 312 Japan) using coupled glycerol 3-phosphate oxidase/peroxidase and cholesterol oxidase/peroxidase photometric tests (Rolf Greiner BioChemica, Wiesenstraße 45, D-65558 Flacht, Germany) with parameter settings as recommended by the reagent manufacturer.

For method comparison, the non-parametrical linear regression procedure of Passing \& Bablok (6) was performed using the statistical data analysis software package EVAPAK (7), obtained by courtesy of Mr. W. Bablok (Boehringer Mannheim GmbH, Sandhofer Straße 116, D-68305 Mannheim, Germany).

Statistical tests for identity of data distribution were performed using the Mann-Whitney U-test procedure.

\section{Results}

\section{Lipid interference in patient samples investigated by method comparison}

Plasma haemoglobin concentrations were determined in 32 heparinate plasma samples with triacylglycerol concentrations ranging from 0.62 to $8.00 \mathrm{mmol} / \mathrm{l}$ (median $=3.47 \mathrm{mmol} / \mathrm{l}$ ) using both the triple wavelength procedure and the ACA plasma haemoglobin method. Plasma haemoglobin concentrations were significantly higher $(p<0.001)$ when measured with the latter method, yielding values of $60(40-100) \mathrm{mg} / \mathrm{l}$ [median (16. -84. percentile)] in comparison to $20(10-30) \mathrm{mg} / 1$ for the triple wavelength method. Furthermore, a poor correlation $(r=0.447, y=2.0 x+10 \mathrm{mg} / \mathrm{l})$ was observed between the haemoglobin concentration values obtained using the two different procedures. Triacylglycerol-rich lipoproteins were then separated from the plasma using an air-driven ultracentrifuge, and plasma haemoglobin concentrations were once again determined with the ACA plasma haemoglobin method. The median plasma haemoglobin concentration value after delipidation was $40(20-50) \mathrm{mg} / \mathrm{l}$ using the ACA method. Although this was still significantly greater $(p<0.005)$ than the value obtained with the comparison method, there was now a good correlation between the ACA method and the triple wavelength method $(\mathrm{r}=0.855, \mathrm{y}=1.0 \mathrm{x}+10 \mathrm{mg} / \mathrm{l})$.

When the values determined with the triple wavelength method were subtracted from those obtained using the ACA plasma haemoglobin procedure and plotted against the respective plasma triacylglycerol concentrations of each sample (fig. 1), substantial positive deviations of the ACA values from the triple wavelength method val- 


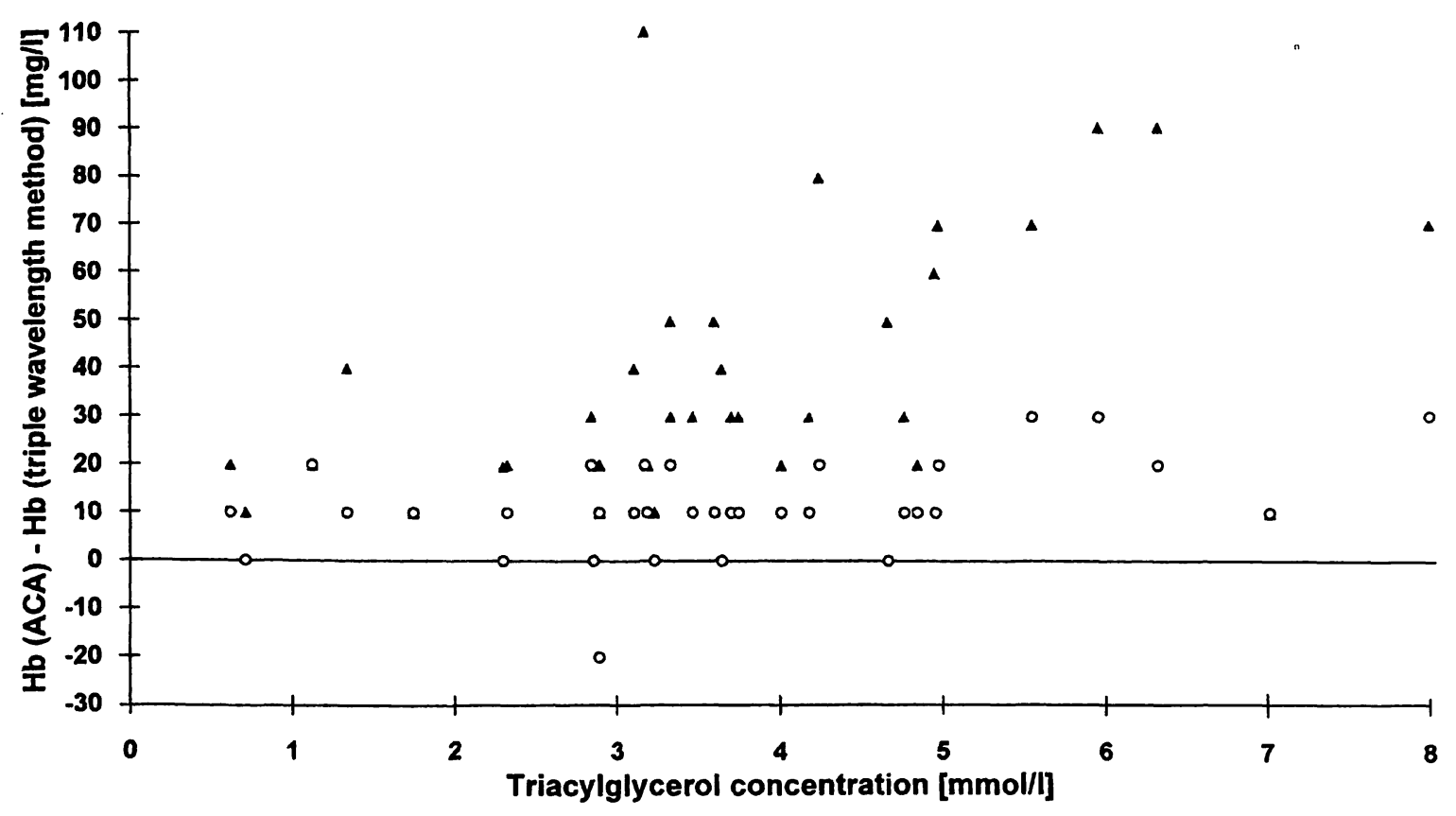

Fig. 1 Difference between the ACA plasma haemoglobin and triple wavelength method haemoglobin in relation to the plasma triacylglycerol concentration.

[४] Untreated patient samples

[o] After airfuge treatment

ues were observed with the native plasma. While there is an apparent trend to increased deviation at elevated triacylglycerol concentrations, there is obviously a wide degree of variation in this deviation even at triacylglycerol concentrations around $3.4 \mathrm{mmol} / \mathrm{l}$.

After separation of the triacylglycerol-rich lipoproteins from the plasma, the ACA plasma haemoglobin method displayed only marginally higher values than the triple wavelength procedure irrespective of the triacylglycerol concentration. We thus achieved a substantial reduction of the overall value differences between the ACA plasma haemoglobin method and the triple wavelength method as indicated by a median (16th -84 th percentile) difference of $10(0-20) \mathrm{mg} / \mathrm{l}$ after delipidation compared with a value of $30(20-70) \mathrm{mg} / \mathrm{l}$ obtained previously. The distribution of the plasma haemoglobin differences for these two populations was significantly different at the $p<0.001$ level. Furthermore, the dependence of the differences between the two methods upon the triacylglycerol concentration decreased after ultracentrifugation: the slope was 15.4 (confidence interval 9.82-23.3) before and 5.04 (confidence interval 2.399.91) after ultracentrifugation.

Influence of triacylglycerol-rich lipoprotein preparations on the ACA plasma haemoglobin procedure

Both VLDL and chylomicrons were isolated from lipaemic plasma by sequential ultracentrifugation. The properties of the isolated human lipoprotein fractions are presented in table 1. As expected, the chylomicron fraction
In each graph, the abscissa represents the triacylglycerol concentration of the native patient plasma sample. The difference between the ACA plasma haemoglobin concentration value and the triple wavelength reference method value is given by the ordinate.

contained particles of a greater size than those in the VLDL fraction.

Increasing amounts of either VLDL or chylomicrons were added to a plasma pool and plasma haemoglobin concentrations were then determined using the ACA procedure. The results were plotted against the respective triacylglycerol concentrations (fig. 2). Addition of increasing amounts of chylomicrons to a normotriglyceridaemic plasma sample pool (triacylglycerol concentration $=0.62 \mathrm{mmol} / \mathrm{l}$ ) led to a linear increase in the ACA plasma haemoglobin concentration value. At a final triacylglycerol concentration of $5.7 \mathrm{mmol} / \mathrm{l}$, the apparent plasma haemoglobin concentration was around $160 \mathrm{mg} / \mathrm{l}$ greater than that of the original sample. In contrast to chylomicrons, addition of VLDL resulted in a much lower interference with the plasma haemoglobin concentration determination. At a final triacylglycerol concentration of $5.7 \mathrm{mmol} / \mathrm{l}$, the ACA plasma haemo-

Tab. 1 Characterization of purified lipoprotein fractions.

\begin{tabular}{lll}
\hline Lipoprotein component & $\begin{array}{l}\text { Chylomicron-rich } \\
\text { preparation }\end{array}$ & VLDL \\
\hline Electrophoresis & $\begin{array}{l}\text { Predominantly } \\
\text { chylomicrons }\end{array}$ & $\begin{array}{l}\text { Predominantly } \\
\text { VLDL }\end{array}$ \\
\hline $\begin{array}{l}\text { Composition } \\
\text { Triacylglycerols (mmol//) }\end{array}$ & 24.1 & \\
Cholesterol (mmol/l) & 12.2 & 21.2 \\
$\begin{array}{l}\text { Particle radius } \\
\text { Mean (SD) (nm) }\end{array}$ & $119(62)$ & 16.8 \\
\hline
\end{tabular}


globin concentration value was only increased by around $30 \mathrm{mg} / \mathrm{l}$ (fig. 2).

Airfuge treatment of samples from either series led to the reduction of the ACA plasma haemoglobin concentration values to concentrations similar to those obtained prior to lipoprotein addition irrespective of the extent of interference observed.

The effect of lipoprotein addition to the plasma pool was analyzed by a non-parametric linear procedure according to Passing \& Bablok (6). The slope from the chylomicron addition experiment of 29.3 (confidence interval 26.3-32.9) was reduced to a value of 3.27 (confidence interval $0.00-8.23$ ) after removal of the triacylglycerol-rich lipoprotein, indicating almost complete elimination of the triacylglycerol interference. The extent of the VLDL interference was much lower, with an initial slope value of 5.40 (confidence interval 3.279.74). After ultracentrifugation, all seven data points yielded an identical haemoglobin concentration of 30 $\mathrm{mg} / \mathrm{l}$ irrespective of the original triacylglycerol concentration. The influence of the two different lipoproteins on the determination of haemoglobin concentrations was also investigated in samples containing normal and elevated plasma haemoglobin concentration levels. The results are presented in figure 3 . The plot shows that chylomicrons in particular can cause substantial interference with the ACA method, relative to the amount of haemoglobin present. In contrast, the presence of VLDL up to triacylglycerol concentrations of $5.7 \mathrm{mmol} / \mathrm{l}$ did not cause a clinically significant lipid interference.
The addition of increasing amounts of chylomicrons to plasma pools containing 30,110 , and $320 \mathrm{mg} / \mathrm{l}$ plasma haemoglobin produced slope values of $30.6,28.7$, and 27.7 (confidence intervals 27.3-33.6, 25.0-33.1, and 18.9-31.2), respectively (fig. 3a). In contrast, VLDL addition with the three plasma haemoglobin concentrations yielded lower slope values of $6.20,4.87$, and 4.51 (confidence intervals 4.69-9.20, 0.00-6.90, and 0.0011.8 ), respectively (fig. $3 \mathrm{~b}$ ). These data confirm the results obtained from figure 2 , showing much greater interference from chylomicrons in comparison to VLDL.

\section{Discussion}

Interferences in clinical chemistry instrumentation are easily visualized in the form of so-called interferographs (8). They offer the potential advantage of arithmetical compensation of the interferant under investigation. With ACA analyzers, a thorough analysis of several interferants with a number of assays has been published (8), however one limitation was that an artificial lipid mixture was used to generate sample turbidity. Furthermore, no published data exist on the effect of lipaemic samples in the ACA plasma haemoglobin procedure.

It is well known that several mechanisms contribute to the observed interference of lipaemia on photometric absorption measurements. One mechanism is dependent on the presence of turbid, light-scattering sample components (3). These light-scattering phenomena are related to particle properties as defined by radius and criteria of

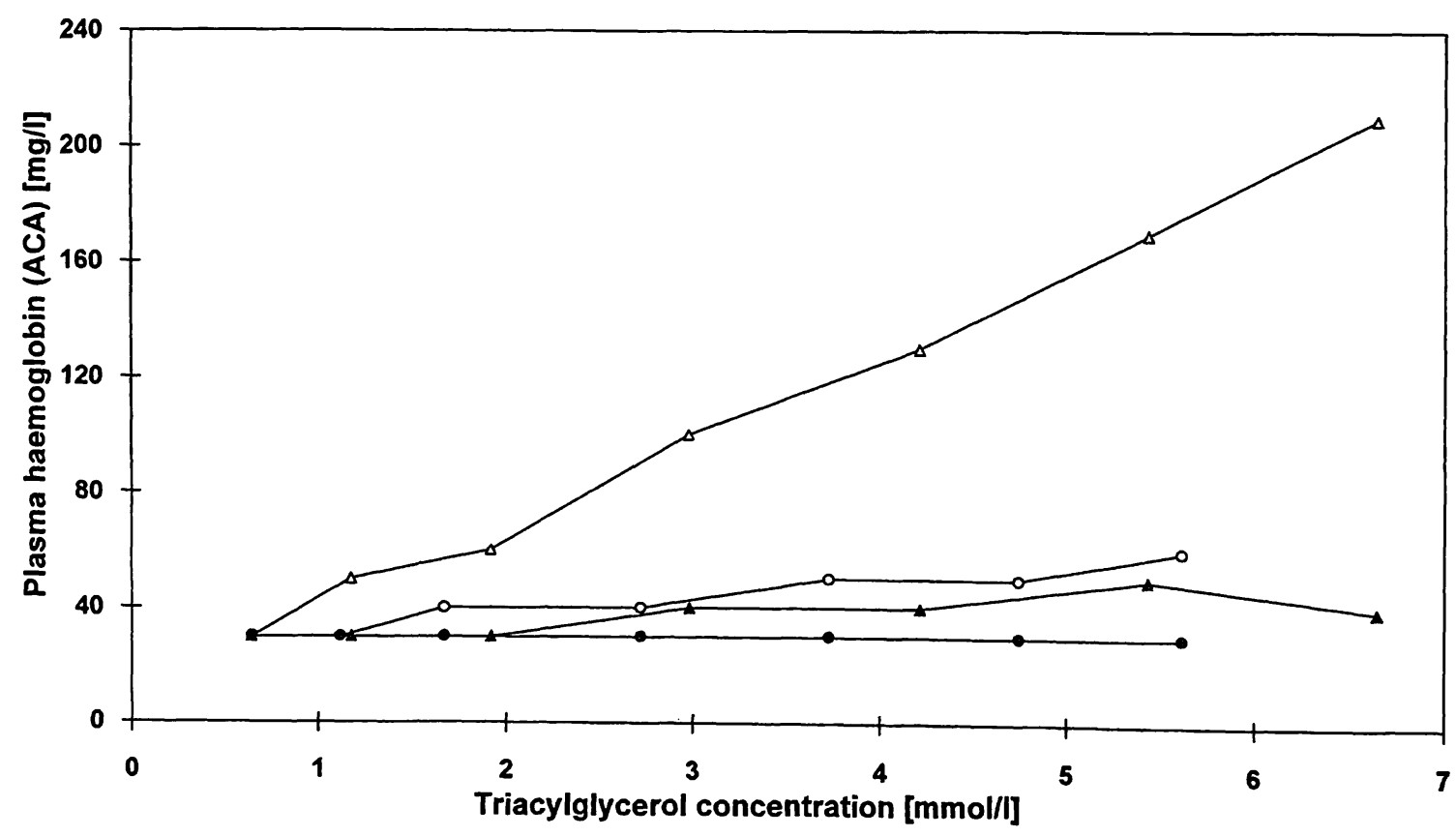

Fig. 2 Influence of supplementation of pool plasma with purified lipoprotein fractions on the ACA plasma haemoglobin method. Purified lipoprotein preparations were added to a pool plasma to

$[-\Delta-]$ Purified human chylomicrons, before delipidation. $y=11.16 \mathrm{mg} / \mathrm{l}+29.3 \cdot x$.

[-А-] Purified human chylomicrons, after delipidation. $y=26.13 \mathrm{mg} / \mathrm{l}+3.27 \cdot x$. give the final triacylglycerol concentration given in the abscissa. ACA plasma haemoglobin values were determined before and after delipidation by airfuge centrifugation.

[-०-] Purified human VLDL, before delipidation. $y=26.52 \mathrm{mg} / \mathrm{l}+5.40 \cdot x$.

[-०-] Purified human VLDL, after delipidation. $y=30 \mathrm{mg} / \mathrm{l}+0.00 \cdot x$. 
shape. It can therefore be surmised that clinical chemical methods relying on photometric measurement will be differentially affected by different lipoprotein subfractions. Using whole blood as the matrix for haemoglobin concentration determinations, differential interference effects of lipoprotein subfractions have been known for a long time (9). The aim of this present investigation was therefore to investigate the influence of lipaemia, and in particular, the effect of different lipoprotein species on the measurement of plasma haemoglobin concentration using the ACA haemoglobin method.

In comparison to a triple wavelength procedure, the ACA method showed considerable interference with hypertriglyceridaemic $(>2.3 \mathrm{mmol} / \mathrm{l})$ plasma. There was, however, substantial variation in the extent of this interference even at similar plasma triacylglycerol concentrations (fig. 1). Since light scattering is proportional to the square of the particle volume (10) and therefore to the sixth power of the radius, it is expected that the size distribution of the lipoprotein particles will be important for the extent of the interference observed. Chylomicrons which are synthesized post-prandially in the intestine are the largest lipoprotein particles. Addition of purified chylomicrons to pool plasma caused considerable interference with the ACA method for the determination of plasma haemoglobin concentration. VLDL is synthesized continuously in the liver and carries endogenously synthesized triacylglycerol as well as cholesterol. Although highly heterogeneous, it is generally of smaller size than the chylomicrons (tab. 1). Purified VLDL was

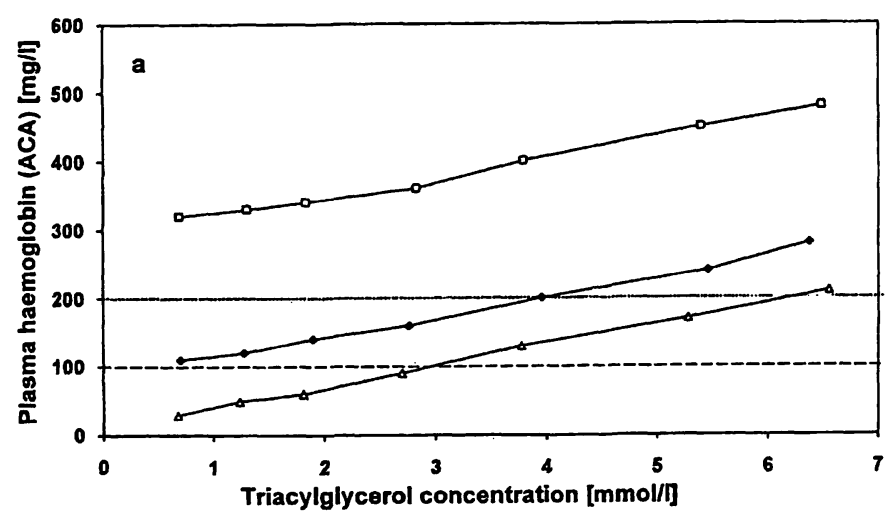

found to have no major effect on the determination of plasma haemoglobin concentration using the ACA method up to a triacylglycerol concentration of 5.7 $\mathrm{mmol} / \mathrm{l}$.

Since preanalytical phenomena (e.g. sampling artifacts) are of major importance for the determination of the plasma haemoglobin concentration, two threshold values have been suggested (11) for clinical evaluation of a single plasma haemoglobin concentration measurement. In addition to the upper reference limit (100 $\mathrm{mg} / \mathrm{l}$ ), an elevated clinical decision value has been recommended, which has to be considered to exclude the presence of extravasal haemolysis. This value is considered to be $200 \mathrm{mg} / \mathrm{l}$ (11). We therefore investigated the influence of triacylglycerol-rich lipoproteins on plasma haemoglobin concentration values in the low reference range, at the upper reference limit of $100 \mathrm{mg} / \mathrm{l}$, and at a pathological plasma haemoglobin concentration. A plasma haemoglobin concentration value of $100 \mathrm{mg} / \mathrm{l}$ will be falsely classified as pathological, that is, above the clinical decision limit of $200 \mathrm{mg} / \mathrm{l}$, at chylomicron triacylglycerol concentrations $>4.0 \mathrm{mmol} / \mathrm{l}$. The influence of VLDL on the determination of plasma haemoglobin concentration values both within the reference range and at pathological values was found to be minimal up to a VLDL triacylglycerol concentration of $5.7 \mathrm{mmol} / \mathrm{l}$.

Furthermore, the statistical evaluation confirms that lipoprotein interference is linear and additive for a specific type of lipoprotein in combination with plasma haemoglobin content.

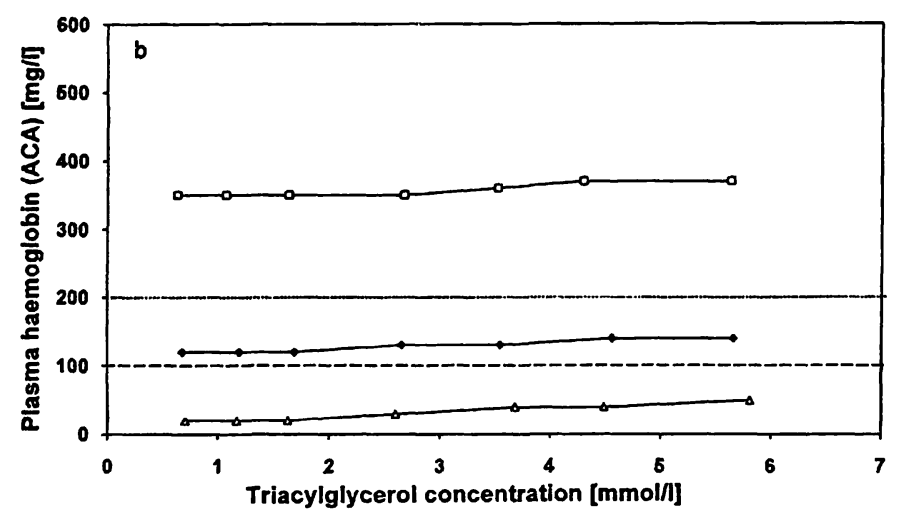

Fig. 3 Influence of triacylglycerol-rich plasma lipoproteins on ACA plasma haemoglobin concentration determination at different haemoglobin values.

a) Purified chylomicrons

$[-\Delta-]$ human plasma pool with a plasma haemoglobin concentration of $30 \mathrm{mg} / \mathrm{l}$. $y=9.23 \mathrm{mg} / \mathrm{l}+30.6 \cdot x$.

[-] human plasma pool supplemented with haemolysate to yield a final plasma haemoglobin concentration of $110 \mathrm{mg} / \mathrm{l}$. $y=85.51 \mathrm{mg} / \mathrm{l}+28.7 \cdot x$.

$[-\square-]$ human plasma pool supplemented with haemolysate to yield a final plasma haemoglobin concentration of $320 \mathrm{mg} / \mathrm{l}$.

$y=295.31 \mathrm{mg} / \mathrm{l}+27.7 \cdot x$.

The dashed line [-----] indicates the upper reference interval, b) Purified VLDL

$[-\Delta-]$ human plasma pool with a plasma haemoglobin concentration of $20 \mathrm{mg} / \mathrm{l}$. $y=13.08 \mathrm{mg} / \mathrm{l}+6.20 \cdot x$.

[-४-] human plasma pool supplemented with haemolysate to yield a final plasma haemoglobin concentration of $120 \mathrm{mg} / \mathrm{l}$. $y=114.21 \mathrm{mg} / 1+4.87 \cdot x$.

[- $\square-]$ Human plasma pool supplemented with haemolysate to yield a final plasma haemoglobin concentration of $350 \mathrm{mg} / \mathrm{l}$. $y=344.33 \mathrm{mg} / \mathrm{l}+4.51 \cdot x$.

the dotted line $[\cdots \cdots \cdot]$ represents the corresponding decision value. 
Based on these results we conclude that the extent of the lipid interference is highly dependent on the lipoprotein species present in the plasma samples. Chylomicrons, but not VLDL, exhibit considerable interference in physiological samples. Plasma haemoglobin concentrations can therefore be correctly determined in non-fasting, chylomicron-containing plasma using the ACA analyzer only after prior delipidation. Several methods have been suggested for the clarification of hyperlipidaemic samples. These include ultracentrifu-

\section{Appendix}

Settings ${ }^{\mathrm{a}}$ for the dual wavelength plasma haemoglobin concentration determination on the ACA SX analyzer [adapted as method \#91]

$\begin{array}{lr}\text { C0 } & -9.470 \\ \text { C1 } & -26.29 \\ \text { P1 } & 1 \\ & \\ \text { P2 } & 13 \\ \text { P3 } & 200 \\ \text { P4 } & 30 \\ \text { P5 } & 10000 \\ \text { P6 } & 0 \\ \text { P7 } & 100 \\ \text { Q1 } & 5 \\ \text { Q2 } & 0 \\ \text { Q3 } & 01 \\ \text { Q4 } & 1750 \\ \text { Q5 } & 4800 \\ \text { R1 } & 0 \\ \text { R2 } & 0000\end{array}$

cf. above

Result is displayed as an integer with no additional decimal place

unit $\mathrm{mg} / \mathrm{l}$

$200 \mu$ l sample volume

lower limit of the assay range

upper limit of the assay range

lower limit of the reference interval

upper limit of the reference interval

first diluent (= glycine buffer)

normal flush

number of flushes

volume of flush

diluent applied to analyzer pack

no second diluent

volume of second diluent

a available on request

\section{References}

1. Golf SW, Schneider S, Friemann E, Temme H, Roka L. Correction of catalytic activities of aspartate aminotransferase, lactate dehydrogenase, acid phosphatase and potassium concentration in haemolytic plasma by determination of haemoglobin concentration with direct spectrophotometry [abstract]. J Clin Chem Clin Biochem 1985, 23:585.

2. Du Pont Produktbereich Diagnostik. aca Methode S HEM (Rev 1-10/95-HG). Bad Homburg; Du Pont de Nemours (Deutschland) $\mathrm{GmbH}, 1995$.

3. McGowan MW, Artiss JD, Zak B. Description of analytical problems arising from elevated serum solids [review]. Anal Biochem 1984, 142:239-51.

4. Fairbanks VF, Ziesmer SC, O'Brien PC. Methods for measuring plasma hemoglobin in micromolar concentrations compared. Clin Chem 1992, 38:132-40.

5. Kahn SE, Watkins BF, Bermes EW. An evaluation of a spectrophotometric scanning technique for measurement of plasma hemoglobin. Ann Clin Lab Sci 1981, 11:126-31.

6. Passing H, Bablok W. A new biometrical procedure for testing the equality of measurements from two different analytical methods. J Clin Chem Clin Biochem 1983, 21:709-20.

7. EVAPAK Prog. V2.30 [computer program], MS-DOS version. Mannheim (Germany): Boehringer Mannheim GmbH Diagnostika, 1993. gation, detergent addition, chemical precipitation, extraction, chromatography, and enzymatic treatment. Most of these involve off-line sample treatment for. separation of lipid constituents, however some methods have been shown to be compatible with the reagents applied in current clinical chemical photometric assays (12). The present data demonstrate that airfuge ultracentrifugation is an effective procedure for removal of lipid interference with the ACA plasma haemoglobin determination method.

$\begin{array}{lrl}\text { R3 } & 0 & \text { preheater on } \\ \text { R4 } & 1 & \text { one incubation cycle } \\ \text { R5 } & 27 & \text { breaker/mixer 1 cycle number } \\ \text { R6 } & 40 & \text { delay breaker/mixer 2 (no delay) } \\ \text { R7 } & 27 & \text { breaker/mixer 2 cycle number } \\ \text { S1 } & 1 & \text { end point determination } \\ \text { S2 } & 10 & \text { suppress time of pack } \\ \text { S3 } & 00 & \text { not in use } \\ \text { S4 } & 09 & \text { first filter } 577 \text { nm } \\ \text { S5 } & 10 & \text { second filter } 600 \mathrm{~nm} \\ \text { S6 } & 01 & \text { number of measurement counts } \\ \text { S7 } & 1 & \text { linear calculation } \\ \text { M1 } & 16 & \text { method name } \\ \text { M2 } & 00 & \text { method name } \\ \text { M3 } & 08 & \text { method name } \\ \text { M4 } & 05 & \text { method name } \\ \text { M5 } & 13 & \text { method name } \\ \text { M6 } & 00 & \text { method name }\end{array}$

8. Glick MR, Ryder KW, Jackson SA. Graphical comparisons of interferences in clinical chemistry instrumentation. Clin Chem 1986, 32:470-5.

9. Gagne C, Auger PL, Moorjani S, Brun D, Lupien P.-J. Effect of hyperchylomicronemia on the measurement of hemoglobin. Am J Clin Pathol 1977, 68:584-6.

10. Hubsch $G$, Houot $O$, Henny J. Influence of turbidity on photometric assays: a blank sample must always be used. J Clin Chem Clin Biochem 1980, 18:149-55.

11. Copeland BE, Dyer PJ, Pesce AJ. Hemoglobin by first derivative spectrophotometry: extent of hemolysis in plasma and serum collected in vacuum container devices. Ann Clin Lab Sci 1989, 19:383-8.

12. Artiss JD, Zak B. Severe hyperlipidemia, an analytical problem: enzymic clearing, a simple solution Trends in Analytical Chemistry 1987, 6:185-91.

Received March 7/July 5, 1996

Corresponding author: Christian Heller, Abteilung Klinische Chemie, Zentrallabor, Georg-August-Universität, Zentrum Innere Medizin, Robert-Koch-Straße 40, D-37075 Göttingen, Germany 